\title{
Combined Influence of Hall Current and Soret Effect on Convective Heat and Mass Transfer Flow past a Vertical Porous Plate in a Rotating Fluid and Dissipation with Convective Boundary Conditions
}

\author{
${ }^{1}$ K. Chandrasekhar Raju and ${ }^{2}$ B. Sreenivasa Reddy \\ ${ }^{1}$ Research scholar in Department of Mathematics, Dravidian University, Kuppam ,A.P \\ ${ }^{2}$ Assistant of Professor, Department of Applied Mathematics, Yogi Vemana University, \\ Kadapa, A.P., India
}

\begin{abstract}
In this Paper, we analyse the effect of thermo-diffusion , and dissipation on convective heat and mass transfer flow of viscous electrically conducting rotating fluid in a vertical rotating plate in the presence of transverse magnetic field under convective boundary conditions with partial slip.. By employing finite element technique the equations governing the flow, heat and mass transfer have been solved. The velocity, temperature and concentration distributions are analysed for different parametric values. The shear stress and rate of heat and mass transfer on the boundary are evaluated numerically for different variations.
\end{abstract}

Keywords : Hall Current, Heat and Mass transfer, Chemical Reaction, Porous Plate, Rotating fluid, Soret effect.

\section{Corresponding Author : K. Chandrasekhar Raju}

\section{INTRODUCTION}

The motion of rotation fluids enclosed with in a body or vice versa, was given by Green span, discussed these problems relating to the boundary layers and their interaction in rotating flows and gave so many examples relating to such interaction. The rotating viscous flow equation yields a layer known as Eckman boundary layer after the Swedish oceanographer Eckman who discovered it. Attempts to observe the structure of the Eckman layer in the surface layers of the sea have been successful. Eckman layers are easy to produce and observe in the laboratory. Such boundary layers or similar ones are required to connect principally geotropic flow in the interior of the fluid to the horizontal boundaries where conditions like a prescribed horizontal stress or no slip on a solid bottom are given. In a similar way other kinds of various boundaries have been studies so as to connect geotropic flow to vertical boundaries (for example a vertical well along which the depth varies) on which boundary conditions consistent with geotropic flow are given. Mahendra Mohan [29] has discussed the free and forced convections in rotating Hydromagnetic viscous fluid between two finitely conduction parallel plates maintained at constant temperature gradients. In view of many scientific and engineering applications of fluids flow through porous media, different authors have been studied [Mahendra Mohan and Srivastava [30], Rao et.al. [39] Sarojamma and Krishna[ 41], Krishna et.al. [27],Seth and Ghosh [43],Agarwal and Dhanpal [5],Ghosh [17],El-Mistikawy et.al. [33a],Hazim Ali Attia [19] the combined free and forced convection flow of an incompressible viscous fluid in a parallel plates channel bounded below by a permeable bed and rotating with a constant angular velocity about an axis perpendicular to the length of the plates 
In the last several years considerable attention has been given to the study of the Hydromagnetic thermal convection due to its numerous applications in geophysics and astrophysics. It is well known that in the geothermal region, gases are electrically conduction and that they undergo the influence of magnetic fluid. Several authors [Gill and Casal [18], Jana [24], Yen [43], Mohanti [35], have theoretically investigated the natural convection effects in forced horizontal flows and considered the effect of wall conductance as convective horizontal channel flow. Circar and Mukherjee [12] have analyzed the effect of mass transfer and rotation on flow past a porous plate in a porous medium with variable suction in a slip flow regime. Balasubramanyam [7] and Reddy [28] have investigated convective heat and mass transfer flow in horizontal rotating fluid under different conditions. Several authors Singh and Mathew [49] Muthucumaraswamy and Ganesan [32], Deka et.al. [15], Muthucumaraswamy [31], Muthucumaraswamy and Meenakshisundaram [28], Chamkha [10], Chamkha [11], Raptis and Perdikis [39], Ibrahim et. al. [20], Indudhar et. al. [21], Cheena Kesavaiah et.al. [11] have studied on oscillatory free convective MHD flow in a rotating vertical porous channel with heat sources.

When heat and mass transfer occur simultaneously in a moving fluid, the relation between the fluxes and the driving potentials are of more intricate nature. Mass fluxes can be created by temperature gradients and this is the Soret effect or thermo-diffusion effect. Several authors Adrian Postelnicu [1], Sreevani et. al. [51], Barletta [8] and Zanchini [56], Barletta [5], Zanchini [56] and Sreevani [51], Sivaiah et. al. [50], Indudhar et. al. [22], Madhusudhan Reddy et. al. [28], Kamalakar et. al. [25], Rajasekhar et. al. [40], Muthucumaraswamy et. al. [33], Jafarunnisa [23], Alam et. al. [6], Srirangavani (52), Jayasudha (58) have studied thermo-diffusion and diffusion thermo effects on combined heat and mass transfer through a porous medium under different conditions. Recently Madhavilatha et al(27a) have discussed the effect of non-linear density-temperature and concentration on rotating convective heat and mass transfer fluid flow past a porous stretching sheet with Soret and Dufour effects. Sukanya et al [57] have discussed combined influence of Hall Currents and Soret effect on convective heat and mass transfer flow past vertical porous stretching plate in rotating fluid and dissipation with constant heat and mass flux and partial slip.

In all these investigations, the effects of Hall currents are not considered. However, in a partially ionized gas, there occurs a Hall current when the strength of the impressed magnetic field is very strong. These Hall effects play a significant role in determining the flow features. Sato [46], Yamanishi [55], Sherman and Sutton [48] have discussed the Hall effects on the steady hydromagnetic flow between two parallel plates. These effects in the unsteady cases were discussed by Pop [30]. Debnath [11] has studied the effects of Hall currents on unsteady hydromagnetic flow past a porous plate in a rotating fluid system and the structure of the steady and unsteady flow is investigated. Alam et. al.,[3] have studied unsteady free convective heat and mass transfer flow in a rotating system with Hall currents, viscous dissipation and Joule heating. Taking Hall effects in to account Krishna et. al.,[27] have investigated Hall effects on the unsteady hydromagnetic boundary layer flow. Rao et. al., [39] have analyzed Hall effects on unsteady Hydromagnetic flow. Siva Prasad et. al., [48a] have studied Hall effects on unsteady MHD free and forced convection flow in a porous rotating channel. Recently Seth et. al., [43] have investigated the effects of Hall currents on heat transfer in a rotating MHD channel flow in arbitrary conducting walls. Sarkar et. al., [44] have analyzed the effects of mass transfer and rotation and flow past a 
porous plate in a porous medium with variable suction in slip flow region. Anwar Beg et al(4) have discussed unsteady magnetohydrodynamics Hartmann-Couette flow and heat transfer in a Darcian channel with Hall current,ionslip,Viscous and Joule heating effects .Ahmed [ 2 ] has discussed the Hall effects on transient flow pas an impulsively started infinite horizontal porous plate in a rotating system. Sukanya et al[57] have investigated the mixed convective heat and mass transfer flow past a porous stretching surface with constant heat and mass flux.

\section{FORMULATION OF THE PROBLEM}

We consider a steady hydromagnetic heat and mass transfer flow of a viscous electrically conducting along a porous infinite vertical plate $\mathrm{y}=0$ in a rotating system. The flow is also assumed to be moving with a uniform velocity $\mathrm{U}_{\infty}$, which is in the $\mathrm{x}$-direction, is taken along the plate in the upward direction and the $y$-axis is normal to it. Initially the plate is at rest, after that the whole system is allowed to rotate with a constant angular velocity $\Omega$ about the y-axis. At the plate are maintained at convective heat and mass boundary conditions. $\mathrm{T}_{\infty}$ and $\mathrm{C}_{\infty}$ are the temperature and species concentration of the uniform flow respectively. A uniform magnetic field $\mathrm{B}$ is taken to be along the $\mathrm{y}-$ axis which is assumed to be electrically non-conducting. We assumed following (Pai [32]) that the magnetic

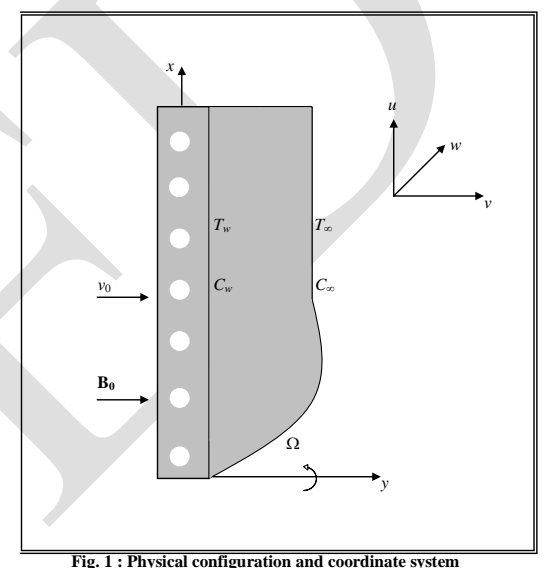

Reynolds number of the flow is taken to be small enough so that the induced magnetic field is negligible in comparison with applied one, so that $\mathrm{B}=(0, \mathrm{~B} 0,0)$ and the magnetic lines of force are fixed relative to the fluid. The equation of conservation of charge $\nabla . \ddot{J}=0$ gives Jy $=$ constant, where the current density $\bar{J}=\left(J_{x}, J_{y}, J_{z}\right)$. Since the plate is electrically nonconducting, this constant is zero and hence $J_{y}=0$ at the plate and also zero everywhere.A uniform magnetic field in the presence of fluid flow induces the current $\left(J_{x}, 0, J_{z}\right)$.

When the strength of the magnetic field is very large we include the Hall current so that the generalized Ohm's law (Cowling[12]) is modified to

$$
\bar{J}+\omega_{e} \tau_{e} \bar{J} x \bar{H}=\sigma\left(\bar{E}+\mu_{e} \bar{q} x \bar{H}\right)
$$

where $\bar{q}$ is the velocity vector. $\mathrm{H}$ is the magnetic field intensity vector. $\bar{E}$ is the electric field, $\bar{j}$ is the current density vector, $\omega_{e}$ is the cyclotron frequency, $\tau_{e}$ is the electron collision time, $\sigma$ is the fluid conductivity and $\mu_{e}$ is the magnetic permeability. The effect of Hall current give rise to a force in the $\mathrm{z}$-direction which in turn produces a cross flow velocity in this direction and thus the flow becomes three -dimensional. To simplify the analysis, we assume that the flow quantities do not vary along z-direction and this will be valid if the surface is of very width along the z-direction. Neglecting the electron pressure gradient, ionslip and thermo-electric effects and assuming the electric field $\mathrm{E}=0$. 
The physical configuration considered here is shown in Figure 1. It is assumed that the plate is semi-infinite in extent and hence all the physical quantities depend on $\mathrm{y}$ and $\mathrm{x}$. Thus accordance with the above assumptions and Boussinesq's approximation, the basic equations relevant to the problem

$$
\begin{aligned}
& \frac{\partial u}{\partial x}+\frac{\partial v}{\partial y}=0 \\
& u \frac{\partial u}{\partial x}+v \frac{\partial u}{\partial y}=v \frac{\partial^{2} u}{\partial y^{2}}+\beta g\left(T-T_{\infty}\right)+\beta^{\bullet} g\left(C-C_{\infty}\right)+2 \Omega w+\frac{\sigma B_{o}^{2}}{\rho}\left(U_{o}-u-m w\right) \\
& u \frac{\partial w}{\partial x}+v \frac{\partial w}{\partial y}=v \frac{\partial^{2} w}{\partial y^{2}}+2 \Omega\left(U_{o}-u\right)-\frac{\sigma B_{o}^{2}}{\rho}\left(m w-\left(u-U_{o}\right)\right) \\
& u \frac{\partial T}{\partial x} v \frac{\partial T}{\partial y}=\frac{k_{f}}{\rho C_{p}} \frac{\partial^{2} T}{\partial y^{2}}+\frac{v}{C_{p}}\left(\left(\frac{\partial u}{\partial y}\right)^{2}+\left(\frac{\partial w}{\partial y}\right)^{2}\right)+ \\
& +\frac{\sigma B_{o}^{2}}{\rho}\left(\left(U_{o}-u\right)^{2}+w^{2}\right) \\
& u \frac{\partial C}{\partial x} v \frac{\partial C}{\partial y}=D_{m} \frac{\partial^{2} C}{\partial y^{2}}+\frac{D_{m} K_{T}}{T_{m}} \frac{\partial^{2} T}{\partial y^{2}}
\end{aligned}
$$

The boundary conditions for the problem are

$$
\begin{aligned}
& u=b x, v=-V_{w}, w=0,-k_{f} \frac{\partial T}{\partial y}=h 1\left(T_{\infty}-T_{w}(x, 0)\right),-D_{m} \frac{\partial C}{\partial y}=h 2\left(C_{\infty}-C_{w}(x, 0)\right) \text { at } \eta=0 \\
& u=U_{o} \quad w=0, T \rightarrow T_{\infty}, C \rightarrow C_{\infty} \quad \text { as } \eta \rightarrow \infty
\end{aligned}
$$

Where $b>0$. The boundary conditions on the velocity in (2.7) are the no-slip conditions at the surface at $\mathrm{y}=0$, while the boundary conditions on the velocity as $y \rightarrow \infty$ follow from the fact that there is no flow far away from the stretching surface. The temperature and species concentration are maintained at a prescribed constant values $\mathrm{T}_{\mathrm{w}}$ and $\mathrm{C}_{\mathrm{w}}$ at the sheet and are assumed to vanish far away from the sheet.

Following the work of Sattar [37], a transformation is now made as

$$
u_{1}=U_{0}-u \quad \Rightarrow u=U_{0}-u_{1}
$$

Equations (2)-(6) and the boundary conditions (6), respectively, transform to

$$
\begin{aligned}
& -\frac{\partial u_{1}}{\partial x}+\frac{\partial v}{\partial y}=0 \\
& \left(U_{0}-u_{1}\right) \frac{\partial u_{1}}{\partial x}+v \frac{\partial u_{1}}{\partial y}=v \frac{\partial^{2} u_{1}}{\partial y^{2}}-\beta g\left(T-T_{\infty}\right)-\beta^{\bullet} g\left(C-C_{\infty}\right)-\frac{\sigma B_{o}^{2}}{\rho}\left(u_{1}-m w\right) \\
& \left(U_{0}-u_{1}\right) \frac{\partial w}{\partial x}+v \frac{\partial w}{\partial y}=v \frac{\partial^{2} w}{\partial y^{2}}+2 \Omega u_{1}-\frac{\sigma B_{o}^{2}}{\rho}\left(m w-u_{1}\right) \\
& \left(U_{0}-u_{1}\right) \frac{\partial T}{\partial x}+v \frac{\partial T}{\partial y}=\frac{k}{\rho C_{p}} \frac{\partial^{2} T}{\partial y^{2}}+\frac{v}{C_{p}}\left(\left(\frac{\partial u_{1}}{\partial y}\right)^{2}+\left(\frac{\partial w}{\partial y}\right)^{2}\right)+ \\
& +\frac{\sigma B_{o}^{2}}{\rho C_{p}}\left(u_{1}^{2}+w^{2}\right)
\end{aligned}
$$


$\left(U_{0}-u_{1}\right) \frac{\partial C}{\partial x}+v \frac{\partial C}{\partial y}=D_{m} \frac{\partial^{2} C}{\partial y^{2}}+\frac{D_{m} k_{T}}{T_{m}} \frac{\partial^{2} T}{\partial y^{2}}-K_{c}^{\prime} C$

$u=b x, v=-V_{w}, w=0,-k_{f} \frac{\partial T}{\partial y}=h 1\left(T_{\infty}-T_{w}(x, 0)\right),-D_{m} \frac{\partial C}{\partial y}=h 2\left(C_{\infty}-C_{w}(x, 0)\right)$ at $\eta=0$

$u=U_{o} \quad w=0, T \rightarrow T_{\infty}, C \rightarrow C_{\infty} \quad$ as $\eta \rightarrow \infty$

Where $\mathrm{u}, \mathrm{v}, \mathrm{w}$ are the velocity components in the $\mathrm{x}, \mathrm{y}, \mathrm{z}$ directions respectively, $v$ is the kinematics viscosity, $\mathrm{g}$ is the acceleration due to gravity, $\rho$ is the density, $\beta$ is the coefficient of Volumetric thermal expansion, $\beta^{*}$ is the Volumetric mass expansion. T, $\mathrm{T}_{\mathrm{w}}$, $\mathrm{T}_{\infty}$ are the temperature of the fluid inside the thermal boundary layer, the plate temperature and the fluid temperature in the free stream respectively, while $\mathrm{C}, \mathrm{C}_{\mathrm{w}}, \mathrm{C}_{\infty}$ are the corresponding concentrations. Also, $\mathrm{K}^{1}$ is the permeability of the porous medium. $\mathrm{k}$ is the thermal conductivity of the medium, $\mathrm{Dm}$ is the coefficient of mass diffusivity, $\mathrm{Cp}$ is the specific heat constant pressure, $\mathrm{Tm}$ is the mean fluid temperature, $\mathrm{k}_{\mathrm{T}}$ is the thermal diffusion ratio, $\mathrm{Cp}$ is the concentration and other symbols have their usual meaning, $\mathrm{C}_{\mathrm{s}}$ is the concentration susceptibility and other symbols have their usual meaning.

\section{MATHEMATICAL ANALYSIS}

In order to solve equations (10)-(13) under the boundary conditions (14), we adopt the welldefined similarity analysis to attain similarity solutions.

For this purpose, the following similarity transformations are now introduced:

$$
\begin{aligned}
& \eta=y \sqrt{\frac{U_{o}}{2 v x}} \\
& g_{o}(\eta)=\frac{w}{U_{o}} \\
& \theta(\eta)=\frac{T-T_{\infty}}{T_{w}-T_{\infty}} \\
& \phi(\eta)=\frac{C-C_{\infty}}{C_{w}-C_{\infty}} \\
& \psi=\sqrt{2 v x U_{0}} f(\eta) \\
& u_{1}=\frac{\partial \psi}{\partial y}=U_{o} f^{\prime}(\eta) \\
& \frac{u}{U_{0}}==1-f^{\prime}(\eta)
\end{aligned}
$$

Now for reasons of similarity, the plate of concentration is assumed to be

$$
C_{w}(x)=C_{o}+\vec{x}\left(C_{0}-C_{\infty}\right)
$$

where $C_{0}$ is considered to be mean concentration and $\vec{x}=\frac{x U_{o}}{v}$

The continuity equation (2) then yields

$$
v=\frac{\partial \psi}{\partial x}=-\sqrt{\frac{\nu U_{o}}{2 x}\left(\eta f^{\prime}(\eta)-f(\eta)\right)}
$$


Also we have $f_{w}=v_{W}(x) \sqrt{\frac{2 x}{v U_{0}}}$

Where $f_{w}$ is the suction parameter or transpiration parameter and clearly in (22) $f_{w}<0$ corresponds to suction and $f_{w}>0$ corresponds to injection at the plate. From equations (10)(14) and (15)-(20), we have the following dimensionless ordinary coupled non-linear differential equations.

$$
\begin{aligned}
& f^{\prime \prime \prime}+(\eta-f) f^{\prime \prime}-G(\theta+N \phi)-D^{-1} f^{\prime}-\frac{M^{2}}{1+m^{2}}\left(f^{\prime}+m g\right)+R g=0 \\
& g^{\prime \prime}+(\eta=f) g^{\prime}-R f^{\prime}+\frac{M^{2}}{1+m^{2}}\left(m f^{\prime}-g\right)=0 \\
& \theta^{\prime \prime}+P_{r}(\eta-f) \theta^{\prime}+\operatorname{Pr} E c\left(\left(f^{\prime 2}\right)+\left(g^{2}\right)\right)+\operatorname{Pr} E c \frac{M^{2}}{1+m^{2}}\left(f^{\prime 2}+g^{2}\right)=0 \\
& \phi^{\prime \prime}+S c(\eta-f) \phi^{\prime}+2 S c f^{\prime} \phi+S c S o \theta^{\prime \prime}=0
\end{aligned}
$$

With the corresponding boundary conditions

$$
\begin{aligned}
& f=f w, f^{\prime}=1+A f^{\prime \prime}(0), g=0, \frac{d \theta}{d \eta}-=-B i(1-\theta(0)), \frac{d \phi}{d \eta}-=-B c(1-\phi(0)), \\
& f^{\prime}=0, \\
& g=0, \theta=0, \phi=0 \quad \text { as } \eta \rightarrow \infty
\end{aligned}
$$

where

$G_{r}=\frac{2 \beta g\left(T_{w}-T_{\infty}\right) x^{3}}{v^{2}}$ (Grashof Number), $N=\frac{\beta^{\bullet}\left(C_{w}-C_{\infty}\right)}{\left(T_{w}-T_{\infty}\right)}$ (Buoyancy parameter),

$D^{-1}=\frac{2 v x}{k U_{0}}$ (Darcy parameter), $M=\frac{2 x \sigma B_{0}^{2}}{\rho U_{0}}$ (Magnetic parameter),

$M_{1}^{2}=\frac{M^{2}}{1+m^{2}}, \quad R=\frac{4 \Omega x}{U_{0}}$ (Rotational parameter), $\quad P_{r}=\frac{\rho v C_{p}}{k}$ (Prandtl Number),

$E c=\frac{U_{0}^{2}}{C_{p}\left(T_{w}-T_{\infty}\right)} \quad($ Eckert Number $), \quad S c=\frac{v}{D_{m}} \quad($ Schmidt $\quad$ Number $), S_{0}=\frac{D_{m} k_{T}\left(T_{w}-T_{\infty}\right)}{T_{m}\left(C_{w}-C_{\infty}\right)}$

(Soret parameter),

For the computational purpose and without loss of generality $\infty$ has been fixed as 8 .The whole domain is divided into 11 line elements of equal width, each element being three nodded.

\section{METHOD OF SOLUTION}

The equations (23 to 26 ) have been solved by employing finite element technique with three nodded approximation functions. The Local Stiffness Matrices have been assembled by using inter element continuity, equilibrium and boundary conditions. The resulting global matrices have been solved by using iteration procedure. The process in continued until the convergence is reached. 


\section{SKIN FRICTION COEFFICIENT, NUSSELT NUMBER AND SHERWOOD NUMBER}

The quantities of chief physical interest are the skin friction coefficients, the Nusselt Number and the Sherwood number. The wall skin frictions are defined by

$$
\begin{aligned}
& \tau_{x}=\mu\left(\frac{\partial u}{\partial y}\right)_{y=0} \quad \text { and } \quad \tau_{z}=\mu\left(\frac{\partial w}{\partial y}\right)_{y=0} \quad \text { which are proportional to } \\
& \left(\frac{\partial^{2} f}{\partial \eta^{2}}\right)_{\eta=0} \text { and }\left(\frac{\partial g_{0}}{\partial \eta}\right)_{\eta=0}
\end{aligned}
$$

$$
\begin{aligned}
& \qquad N u=\frac{1}{\Delta T}\left(\frac{\partial T}{\partial y}\right)_{y=0} \text { which is proportional to }\left(\frac{\partial \theta}{\partial \eta}\right)_{\eta=0} \\
& \text { The Nusselt Number is defined by } \\
& \text { The Sherwood Number is defined by } S h=\frac{1}{\Delta C}\left(\frac{\partial C}{\partial y}\right)_{y=0} \text { which is proportional to }\left(\frac{\partial \phi}{\partial \eta}\right)_{\eta=0}
\end{aligned}
$$

The numerical values of the skin friction coefficients, the Nusselt Number and the Sherwood Number are sorted in table .2 .

\section{COMPARISON}

In the absence of Hall currents $(\mathrm{m}=0)$ and no slip regime $(\mathrm{A}=0)$ and $\mathrm{Bi}=\mathrm{Bc}=1$, the results are in good agreement with Sreerangavani et al[52].

Table. 1 shows the comparison

\begin{tabular}{|l|l|l|l|l|l|l|l|l|}
\hline \multicolumn{3}{|l|}{ Parameters } & \multicolumn{3}{l|}{ Sreerangavani et al[52] } & \multicolumn{3}{l|}{ Present results $(\mathrm{m}=0, \mathrm{~A}=0, \mathrm{Bi}=\mathrm{Bc}=1)$} \\
\hline $\mathrm{R}$ & So & $\mathrm{Ec}$ & $\tau \mathrm{x}(0)$ & $\mathrm{Nu}(0)$ & $\mathrm{Sh}(0)$ & $\tau \mathrm{x}(0)$ & $\mathrm{Nu}(0)$ & $\mathrm{Sh}(0)$ \\
\hline 0.5 & 0.5 & 0.01 & 3.49064 & 0.07426 & 1.2087 & 3.48997 & 0.07429 & 1.2091 \\
\hline 1.0 & 0.5 & 0.01 & 3.48376 & $\mathbf{0 . 0 7 3 2 8}$ & 1.2079 & 3.48372 & $\mathbf{0 . 0 7 3 2 9}$ & 1.2081 \\
\hline 1.5 & 0.5 & 0.01 & 3.47259 & $\mathbf{0 . 0 7 1 6 4}$ & 1.2054 & 3.47261 & $\mathbf{0 . 0 7 1 6 9}$ & 1.2052 \\
\hline 0.5 & 1.0 & 0.01 & 3.48853 & 0.07523 & 1.2761 & 3.48849 & 0.07521 & 1.2762 \\
\hline 0.5 & 1.5 & 0.01 & 3.48704 & 0.07596 & 1.3214 & 3.48709 & 0.07599 & 1.3210 \\
\hline 0.5 & 0.5 & 0.03 & 3.43136 & 0.08732 & 1.4987 & 3.43133 & 0.08736 & 1.4989 \\
\hline 0.5 & 0.5 & 0.05 & 3.57222 & 0.05684 & 0.8670 & 3.57221 & 0.05686 & 0.8669 \\
\hline 0.5 & 0.5 & 0.07 & 3.69426 & 0.03131 & 0.4445 & 3.69424 & 0.03128 & 0.4448 \\
\hline
\end{tabular}

\section{DISCUSSION OF THE NUMERICAL RESULTS}

The non-linear linear equations governing the flow have been analysed by employing Galerkin finite element technique with three nodded line segments. The velocity,temperature and concentrations distributions have been analysed for different variations of the parameters $\mathrm{m}, \mathrm{R}, \mathrm{So}, \mathrm{Ec}, \mathrm{Bi}$ and Bc.

Figs.2-7 show the variation of the axial velocity $f^{1}(\eta)$ with different values of $m, R$, So, $\mathrm{Ec}, \mathrm{Bi}$ and $\mathrm{Bc}$. An increase in the Hall parameter(m) decreases the axial velocity in the flow region(fig.2a). Fig.6a represent $f^{1}(\eta)$ with rotation parameter R(fig.3). It can observed from the profiles that $f^{1}(\eta)$ reduces with increase in the rotation parameter R. Increasing the Soret parameter $S_{0}$ larger the axial velocity in the flow region (fig.4a). With reference to Ec(Fig.5), it can be seen that higher the dissipative heat larger the velocity. From figs.6a\&7a we find 
that the axial velocity reduces with increase in the convective heat and mass transfer constants Bi\&Bc.

The cross velocity $(g(\eta))$ which arises due to the rotation and hall current is shown in figures $2 b-7 b$ for different parametric values. It is found that the cross velocity $g(\eta)$ enhances with increase in $\mathrm{m}$, Ec,So (figs. $2 \mathrm{~b}, 4 \mathrm{~b}, 5 \mathrm{~b}$ ) and reduces with inverse rotation parameter (R),Soret parameter (So) and Eckert number (Sc). When the molecular buoyancy force dominates over the thermal buoyancy force the magnitude of cross velocity reduces when the buoyancy forces are in the same direction and for the forces acting in opposite directions. The cross velocity reduces with $\mathrm{Bi}$ and enhances with increase in $\mathrm{Bc}$ (figs.6b\&7b).

The non-dimensional temperature $(\theta)$ is shown in figures $2 c-7 c$ for different parametric values. Higher the Hall parameter (m) /rotation parameter(R) larger the temperature in the flow region (figs.2c,3c). Increasing the Soret parameter $S_{0}$ results in an enhancement in the temperature (fig.4c). An increase in Eckert number Ec leads to an enhancement in the temperature (fig.5).From figs.6c\&7c we find that higher the convective heat and mass transfer constants larger the temperature in the flow region.

The concentration distribution $(\mathrm{C})$ is shown in figures.2d-7d for different parametric values. We follow the convention that the non-dimensional concentration is positive/negate according as actual concentration is greater/lesser than the ambient concentration. It is found that an increase in $\mathrm{m}$ leads to a depreciation in the actual concentration (figs. 2d). Increasing Soret parameter $S_{0}$ leads to an enhancement in the concentration (fig.4d). From fig.5, we find that the concentration reduces with Eckert number Ec. An increase in rotation parameter $\mathrm{R}$ leads to an enhancement in the concentration (fig.3d).
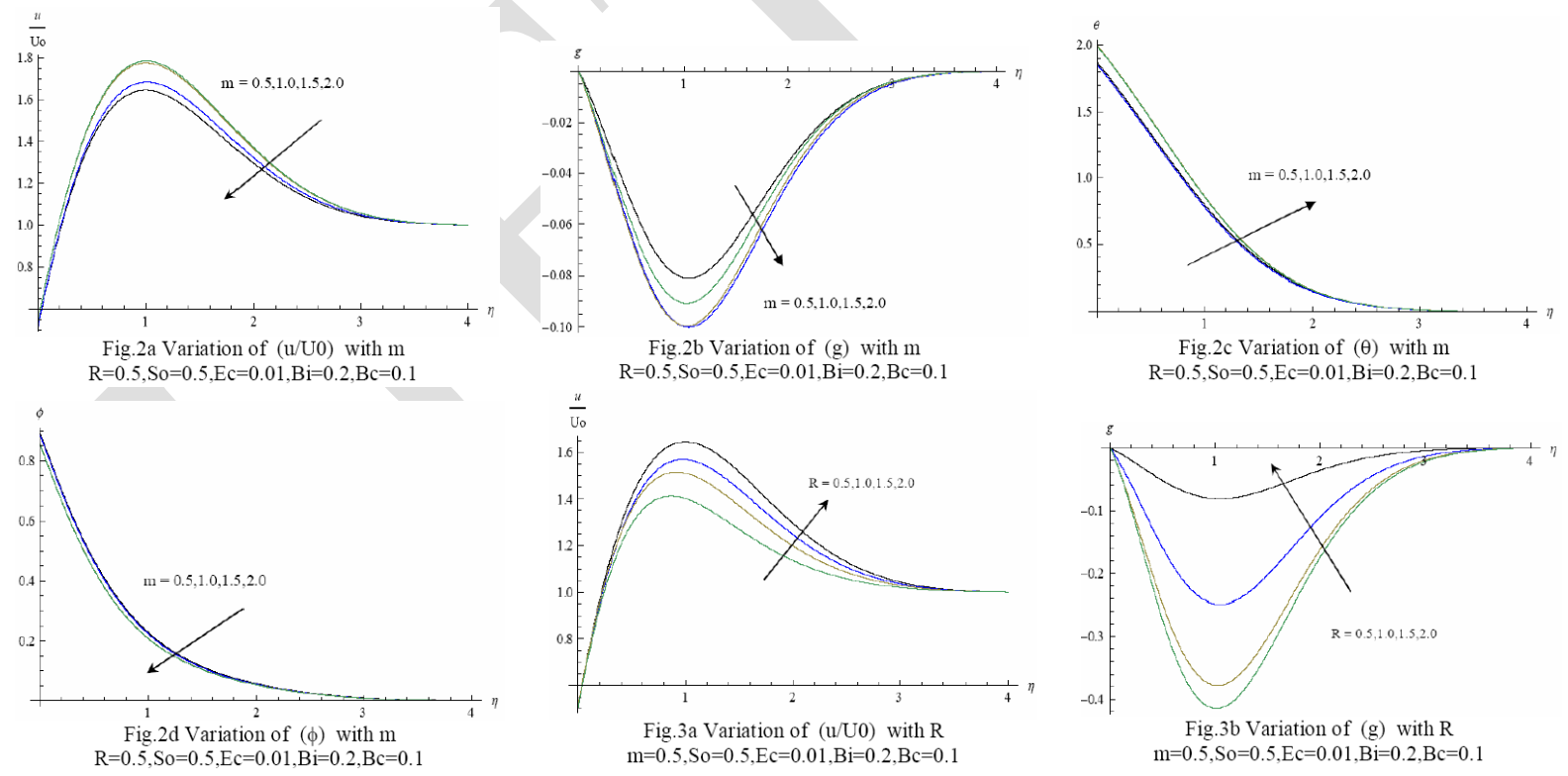
DOI : https://dx.doi.org/10.26808/rs.ed.i9v3.03

International Journal of Emerging Trends in Engineering and Development Available online on http://www.rspublication.com/ijeted/ijeted_index.htm
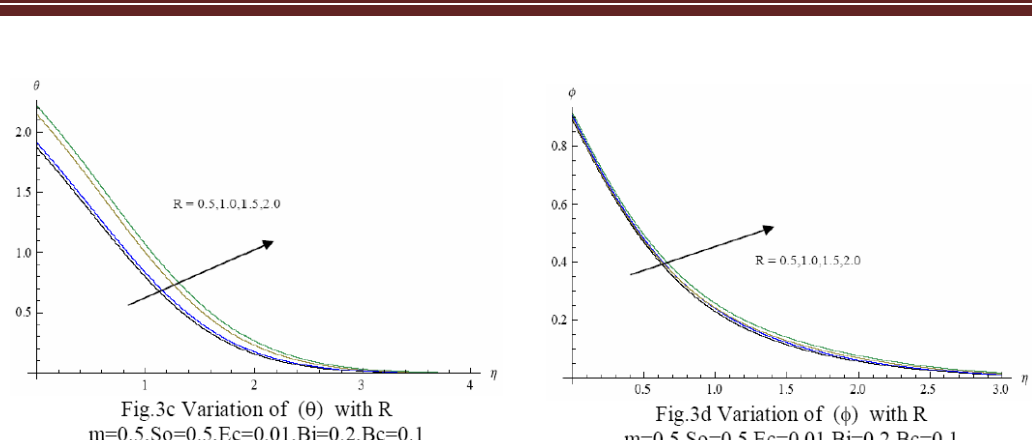
$\mathrm{m}=0.5, \mathrm{So}=0.5, \mathrm{Ec}=0.01, \mathrm{Bi}=0.2, \mathrm{Bc}=0$
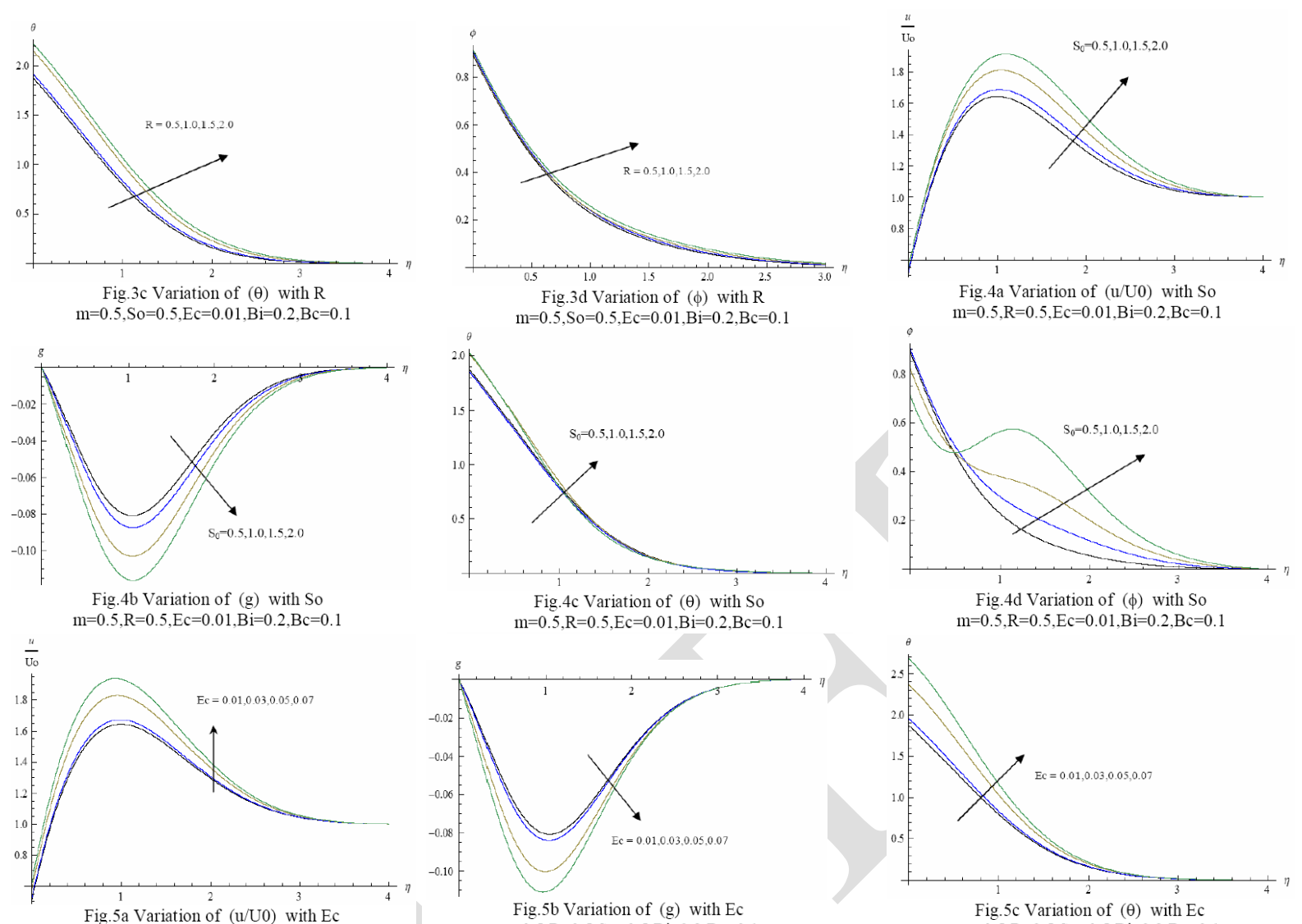

$\mathrm{m}=0.5, \mathrm{R}=0.5$, $\mathrm{m}=0.5, \mathrm{R}=0.5, \mathrm{So}=0.5, \mathrm{Bi}=0.2, \mathrm{Bc}=0.1$
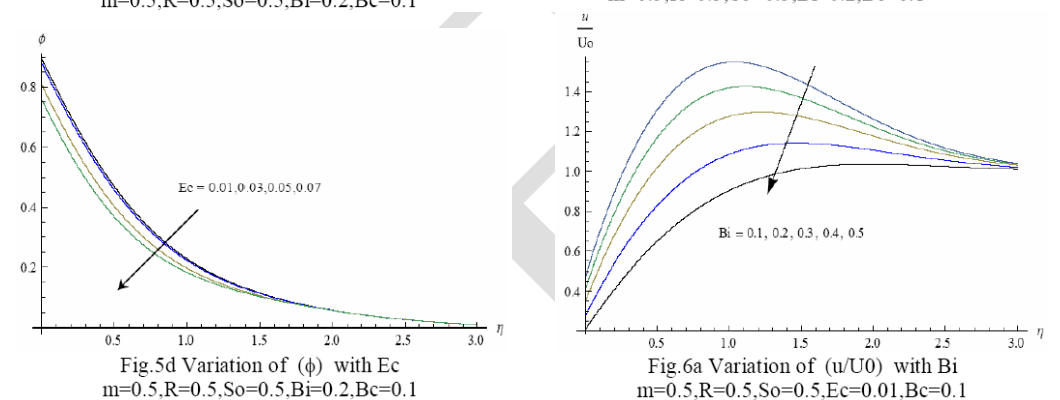

Fig $4 \mathrm{~d}$ Variation of $(\phi)$ with So $\mathrm{m}=0.5, \mathrm{R}=0.5, \mathrm{Ec}=0.01, \mathrm{Bi}=0.2, \mathrm{Bc}=0.1$

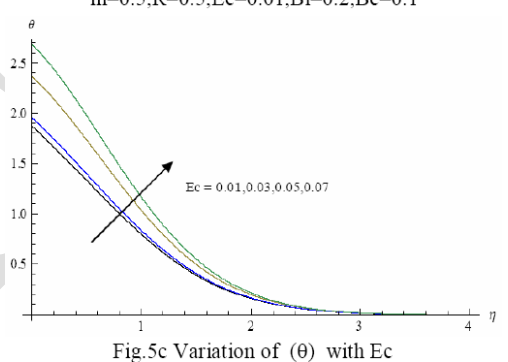
$\mathrm{m}=0.5, \mathrm{R}=0.5, \mathrm{So}=0.5, \mathrm{Bi}=0.2, \mathrm{Bc}=0.1$

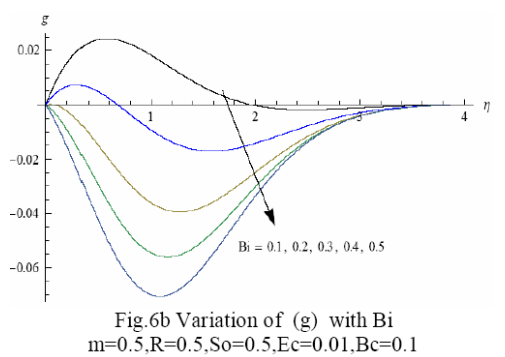


DOI : https://dx.doi.org/10.26808/rs.ed.i9v3.03
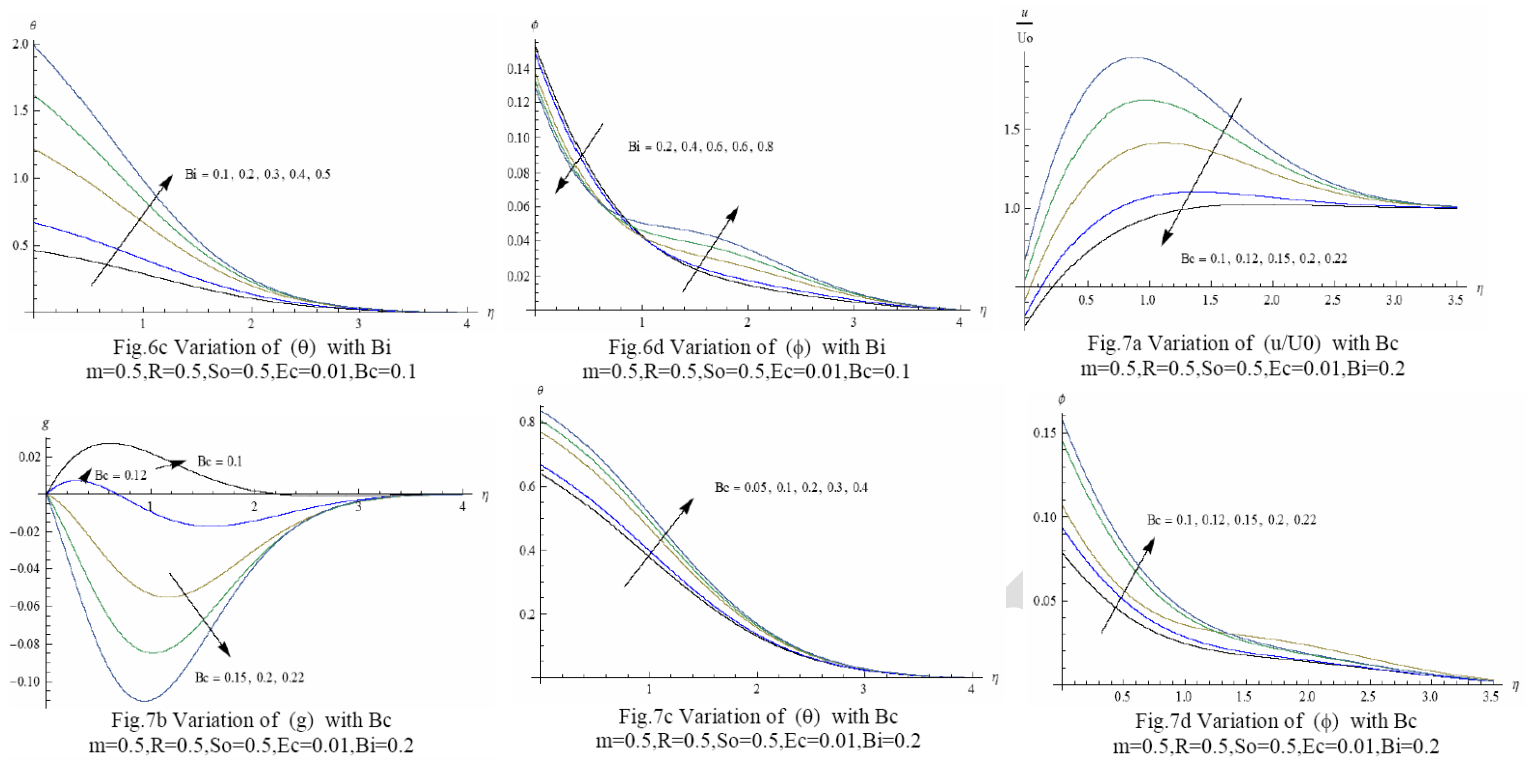

Table 1 : Skin friction $(\tau)$, Nusselt Number $(\mathrm{Nu})$ at $\eta=0$

\begin{tabular}{|cc|c|c|c|c|}
\hline \multicolumn{2}{|c|}{ Parameter } & $\boldsymbol{\tau}_{\boldsymbol{x}}(\mathbf{0})$ & $\boldsymbol{\tau}_{\boldsymbol{z}}(\mathbf{0})$ & $\mathbf{N u}(\mathbf{0})$ & $\mathbf{S h}(\mathbf{0})$ \\
\hline $\mathbf{m}$ & $\mathbf{0 . 5}$ & -2.59033 & -0.079692 & 0.534913 & 1.1203 \\
& $\mathbf{1 . 0}$ & -2.60106 & -0.102789 & 0.539857 & 1.12706 \\
& $\mathbf{1 . 5}$ & -2.72838 & -0.111423 & 0.540852 & 1.16808 \\
& $\mathbf{2 . 0}$ & -2.73211 & -0.121966 & 0.551873 & 1.16965 \\
\hline $\mathbf{R}$ & $\mathbf{0 . 5}$ & -2.59033 & -0.079692 & 0.534913 & 1.1203 \\
& $\mathbf{1 . 0}$ & -2.628413 & -0.120678 & 0.533361 & 1.13811 \\
& $\mathbf{1 . 5}$ & -2.69052 & -0.185135 & 0.490127 & 1.15165 \\
& $\mathbf{2 . 0}$ & -2.65772 & -0.282248 & 0.482666 & 1.14009 \\
\hline So & $\mathbf{0 . 5}$ & -2.59033 & -0.079692 & 0.534913 & 1.1203 \\
& $\mathbf{1 . 0}$ & -2.64864 & -0.0983021 & 0.548881 & 1.0827 \\
& $\mathbf{1 . 5}$ & -2.75688 & -0.126451 & 0.593793 & 1.00159 \\
& $\mathbf{2 . 0}$ & -2.77041 & -0.138365 & 0.680923 & 0.99381 \\
\hline Ec & $\mathbf{0 . 0 1}$ & -2.59033 & -0.079692 & 0.534913 & 1.12033 \\
& $\mathbf{0 . 0 3}$ & -2.64414 & -0.086250 & 0.512556 & 1.13957 \\
& $\mathbf{0 . 0 5}$ & -2.82623 & -0.109015 & 0.452638 & 1.20124 \\
& $\mathbf{0 . 0 7}$ & -4.12237 & -0.253574 & 0.241422 & 1.77627 \\
\hline Bi & $\mathbf{0 . 1}$ & -2.59033 & -0.079692 & 0.534913 & 1.1203 \\
& $\mathbf{0 . 2}$ & -2.03456 & -0.085643 & 0.556754 & 1.0245 \\
& $\mathbf{0 . 3}$ & -1.67543 & -00956433 & 0.589765 & 0.9854 \\
& $\mathbf{0 . 4}$ & -1.45676 & -0.123454 & 0.603456 & 0.9235 \\
\hline Bc $\mathbf{0 . 0 5}$ & -2.59033 & -0.079692 & 0.534913 & 1.1203 \\
$\mathbf{0 . 1 0}$ & -1.56785 & -0.065455 & 0.556746 & 1.2435 \\
$\mathbf{0 . 1 5}$ & -1.23457 & -0.556743 & 0.576548 & 1.3056 \\
$\mathbf{0 . 2 0}$ & -0.96754 & -0.045666 & 0.586643 & 1.4056 \\
\hline & & & & & \\
\hline
\end{tabular}

The components of skin friction $\tau_{\mathrm{x}} \& \tau_{\mathrm{z}}$ are depicted in table 2 for different values of $\mathrm{m}, \mathrm{R}, \mathrm{Ec}, \mathrm{S}_{0}, \mathrm{Bi}$ and $\mathrm{Bc}$. It is found that $\tau_{\mathrm{x}} \& \tau_{\mathrm{z}}$ enhances with increase in $\mathrm{m}$. $\tau_{\mathrm{z}}$ enhances at 
the wall. $\tau_{\mathrm{x}}$ reduces with increase in convective heat ad mass transfer constants $\mathrm{Bi} \& \mathrm{Bc}$ at the wall while $\tau_{\mathrm{z}}$ enhances with $\mathrm{Bi}$ and reduces with $\mathrm{Bc}$ at $\eta=0$. Higher the dissipative heat larger $\tau_{\mathrm{x}} \& \tau_{\mathrm{z}}$ at the wall. It can be seen that increasing the Soret parameter $\mathrm{S}_{0}$ results in an enhancement in $\tau_{\mathrm{x}}$ and $\tau_{\mathrm{z}}$ at the wall $\eta=0$.

The rate of heat transfer (Nusselt number) at $\eta=0$ is exhibited in table 2 for different parametric values. Higher the thermal buoyancy force larger the rate of heat transfer at the wall $\eta=0$. An increase in the Hall parameter $m$ enhances the rate of heat transfer at the wall. $|\mathrm{Nu}|$ reduces with increase in the rotation parameter $\mathrm{R}$ or Eckert number Ec. The rate of heat transfer at the wall experiences an enhancement with increase in the convective heat and mass transfer constants Bi\&Bc. For an increase in the Soret parameter So, we notice an enhancement in $\mathrm{Nu}$ at the wall.

The rate of mass transfer (Sherwood number) at $\eta=0$ is shown in table 2 for different parametric values. It is found that the rate of mass transfer enhances with increase in $\mathrm{m}$. $|\mathrm{Sh}|$ reduces with increase in the rotation parameter $\mathrm{R}$ and enhances with increase in Ec. Increasing the Soret parameter $\mathrm{S}_{0}$ leads to a depreciation in the rate of mass transfer at the wall The rate of mass transfer at the wall reduces with $\mathrm{Bi}$ and enhances with $\mathrm{Bc}$.

\section{CONCLUSIONS}

An attempt has been made to discuss the combined impact of rotation and Hall currents on convective heat and mass transfer flow of a viscous fluid through a porous medium past a stretching surface. Using Finite element technique the governing equations have been solved .The important conclusion of this analysis are

$>$ The profiles the primary and secondary velocities enhance, the temperature and concentration reduces with increase in $\mathrm{G}$. The stress components $\tau \mathrm{x}$, $\tau \mathrm{Z}$, Nusselt and Sherwood number enhances with G.

$>$ An increase in Hall parameter reduces the primary velocity, concentration, enhances the secondary velocity, concentration. The stress components, Nusselt and Sherwood number enhances with $\mathrm{m}$.

$>$ An increase in rotation parameter $(\mathrm{R})$ enhances the primary velocity, temperature, concentration and reduces the secondary velocity in the flow region. The Nusselt number reduces and Sherwood number enhances at the wall with rotation parameter $\mathrm{R}$.

$>$ The effect of thermo-diffusion is to enhance the velocities, concentration, temperature. The stress components,temperature enhances while the concentration reduces with So.

$>$ An increase in the slip parameter (A) enhances the primary velocity,reduces the secondary velocity,temperature and concentration in the flow region.the Nusselt and Sherwood number enhances on the wall.

$>\mathrm{An}$ increase in $\mathrm{Bi}$ and $\mathrm{Bc}$ reduces the primary velocity,and enhances the secondary velocity and temperature while the concentration reduces with $\mathrm{Bi}$ and enhances with $\mathrm{Bc}$.The rate of heat and mass transfer enhance on the wall with increase in $\mathrm{Bi}$ and $\mathrm{Bc}$.

\section{REFERENCES}

1] Adrian Postelnicu : Influence of magnetic field on heat and mass transfer by natural convection from vertical surfaces in porous media considering Soret and Dufour effects. Int. J. of Heat and Mass Transfer, V.47, pp.1467-1472 (2004). 
2] Ahmed $N$ and H.K. Sarmah : MHD Transient flow past an impulsively started infinite horizontal porous plate in a rotating system with hall current: Int J. of Appl. Math and Mech. 7(2) : 1-15, 2011.

3] Alam, M.M and Sattar,M.A :Unsteady free convection and mass transfer flow in a rotating system with Hall currents, viscous dissipation and Joule heating ., Joural of Energy heat and mass transfer,V.22,pp.31-39(2000)

4] Anwar Beg, O,Joaquin Zueco and Takhar,H.S:Unsteady magneto-hydrodynamic Hartmann-Couette flow and heat transfer in a Darcian channel with hall currents, ionslip, Viscous and Joule heating:Network Numerical solutions, Commun Nonlinear Sci Numer Simulat,V.14,pp.1082-1097(2009).

5] Agarwal, R.S and Dhanapal, C : Numerical solution to the flow of a micro polar fluid flow through porous walls of different permeability. pp. 325-336 (1987).

6] Alam, Md, Delower Hossain, $M$ and Arif Hossain, $M$ : Viscous dissipation and joule heating effects on steady MHD combined heat and mass transfer flow through a porous medium in a rotating system. Journal of Naval Architecture and Marine Engineering, V.2, pp.105-120 (2011).

7] Balasubramanyam $M$ : Effect of radiation on convective Heat and Mass transfer flow in a horizontal rotating channel communicated to Research India Publications, India (2010).

8] Barletta, A : Laminar mixed convection with viscous dissipation in a vertical channel. Int. J. Heat Mass Transfer, V.41, PP.3501-3513 (1998).

9] Brewester, M.Q : Thermal radiative transfer and properties. John Wiley \& Sons. Inc. NewYork (1992).

10] Chamkha, A.J : MHD flow of a numerical of uniformly stretched vertical permeable surface in the presence of heat generation/absorption and a chemical reaction. Int. Comm. Heat Mass transfer, V.30, pp.413-422 (2003).

11] Chenna Kesavaiah, D , Satyanarayana, P.V and Venkataramana, S : Effects of the chemical reaction and radiation absorption on an unsteady MHD convective heat and mass transfer flow past a semi-infinite vertical permeable moving plate embedded in a porous medium with heat source and suction. Int. J. of Appl. Math. \& Mech., V.7, No.1, pp.52-69 (2011).

12] Circar and Mukharjee : Effects of mass transfer and rotation on flow past a porous plate in a porous medium with variable suction in slip flow. Acta Cienica Indica, V.34M, No.2, pp.737-751 (2008).

13] Debnath,L:Exact solutions of unsteady hydrodynamic and hydromagnetic boundary layer equations in a rotating fluid system, ZAMM, V.55, p.431 (1975)

14] Debnath,L:ZAMM.V.59,pp.469-471(1979)

15] Deka, R, Das, U.N, Soundalgekar, V.M : Effects of mass transfer on flow past an impulsively started infinite vertical plate with constant heat flux and chemical reaction. Forschung in Ingenieurwesen, V.60, pp284-287 (1994).

16] El.Mistikawy, T.M.A, Attia, H.A : The rotating disk flow in the presence of Strong magnetic field. Proc. $3^{\text {rd }}$ Int. Congr. of fluid mechanics. Cairo, Egypt. V.3, 2-4 January, pp 1211-1222 (1990). 
17] Ghouse, S.K : A note on steady and unsteady hydro magnetic flow in rotating channel in the presence of inclined magnetic field. Int. J. Eng. Sci., V.29, No.8, pp.10131016(1991).

18] Gill, W.M, and Casal, A.D : A theoretical investigation of natural convection effects in forced horizontal flows. Amer. Inst. Chem. Eng. Jour., V.8, pp.513-520(1962).

19] Hazem Ali Attia : Unsteady MHD flow near a rotating porous disk with uniform suction or injection. Fluid dynamics Research, V.23, pp.283-290.

20] Ibrahim, F.S, Elaiw, A.M and Bakr, A.A : Effect of chemical reaction and radiation absorption on unsteady MHD free convection flow past a semi-infinite vertical permeable moving plate with heat source and suction. Comm. In Non-linear Sci. and Num. Simulation, V.13, pp.1056-1066 (2008).

21] Indudhar Reddy, M, Narasimha Rao, $P$ and Prasada Rao, D.R.V : Effect of chemical reaction and radiation absorption on unsteady MHD double diffusive convective flow of a viscous fluid past a semi-infinite porous plate. J. Pure \& Appl. Phys., V.23, No.2, pp.205-215 (2011).

22] Indudhar Reddy, M, Narasimha Rao, $P$ and Prasada Rao, D.R.V : Effect of Quadratic density-temperature variation on unsteady convective heat and mass transfer flow in a vertical channel. J. Pure \& Appl. Phys., V.23, No.2, pp.175-187 (2011).

23] Jafarunnisa, $S$ : Transient double diffusive flow of a viscous fluid with radiation effect in channels/Ducts, Ph.D Thesis, S.K.University, Anantapur, India (2011).

24] Jana, R.N, Angew, A : Wall conductance effects on convective horizontal channel flow. Math and Phys., V.26, pp.315-324 (1975).

25] Kamalakar, P.V.S, Prasada Rao, D.R.V : Finite element analysis of chemical reaction effect on non-darcy convective heat $\&$ mass transfer flow through a porous medium in vertical channel with heat sources. Int. j. Appl. Math \& Mech., V.13, pp.13-28(2012).

26] Krishna,D.V and Prasada ra;,D.R.V :Hall effects on the unsteady hydrmagnetic boundary layer flow .,Acta Mechanica,V.30,pp.303-309 (1981)

27] Krishna,D.V,Prasada rao,D.R.V,Ramachandra Murty,A.S:Hydromagnetic convection flow through a porous medium in a rotating channel., J.Engg. Phy. and Thermo.Phy,V.75(2),pp.281-291(2.

27a] Madhavilatha,S and Prasada rao,D.R.V: Finite element analysis of convective heat and mass transfer flow past a vertical porous plate in a rotating fluid.,Int.Jour.Emerging and development,Vol.3,pp.202-216,(2017)

28] Madhusudhan Reddy, Y, Prasada Rao, D.R.V : Effect of thermo diffusion and chemical reaction on non-darcy convective heat $\&$ mass transfer flow in a vertical channel with radiation. IJMA, V.4, pp.1-13 (2012).

29] Mohan, $M$ : Combined effects of free and forced convection on magneto hydrodynamic flow in a rotating channel. Proc. Indian Acad. Sc., V.85, pp.383-401 (1977)

30] Mohan, M, Srivatsava, K.K : Combined convection flows through a porous channel rotating with angular velocity. Proc. Indian Acad. Sci., V.87, p.14 (1978).

31] Muthucumaraswamy, $R$ : Effects of a chemical reaction on a moving isothermal surface with suction. Acta Mechanica, V.155, pp.65-72 (2002).

32] Muthucumaraswamy, $R$ and Ganesan, $P$ : Effect of the chemical reaction and injection on flow characteristics in an unsteady upward motion of an isothermal plate. J. App. Mech. Tech. Phys., V.42, pp.665-671 (2001). 
33] Muthucumaraswamy, $\mathrm{R}$, Dhanasekhar, $\mathrm{N}$ and Eswar Prasad, G : Rotation effects on flow past an accelerated isothermal vertical plate with chemical reaction of first order. IJMA, V.3, No.5, pp.2122-2129 (2012).

34] Muthucumaraswamy, $\mathrm{R}$ and Meenakshisundaram, S : Theoretical study of chemical reaction effects on vertical oscillating plate with variable temperature. Theoret. Appl. Mech., V.33, pp.245-257 (2006).

35] Nanda R.S and Mohanty, H.K : Hydromagnetic flow in a rotating channel. Appl. Sci. Res., V.24, pp.65-78(1970).

36] Pai, S.I : Magnetogasdynamics and Plasma dynamics. Springier Verlag, New York (1962).

37] Prasada Rao, D.R.V, Krishna, D.V and Debnath, L : Combined effect of free and forced convection on MHD flow in a rotating porous channel. Int. J. Math and Math. Sci., V.5, pp.165-182 (1982).

38] Rajasekhar, N.S, Prasad, P.M.V and Prasada Rao, D.R.V : Effect of Hall current , Thermal radiation and thermo diffusion on convective heat and mass transfer flow of a viscous, rotating fluid past a vertical porous plate embedded in a porous medium. Advances in Applied Science Research, V.3, No.6, pp.3438-3447 (2012).

39] Raptis A, Perdikis $C$ : Free convection flow of water near $4 \mathrm{C}$ past a moving plate. Forschung in Ingenieurwesen, V. 67, pp.206-208 (2002).

40] Rao,D.R.V and Krishna,D.V :Hall effects on unsteady hydromagnetic flow., Ind.J.Pure and Appl.Maths,V.12(2),pp.270-276(1981)

41] Sarojamma, G and Krishna, D.V : Transient Hydromagnetic convection flow in a rotating channel with porous boundaries. Acta Mechanica, V. 39, p.277 (1981).

42] Sattar, M.A : Free and forced convection boundary layer flow through a porous medium with large suction. Int. J. of Energy Research, V.17, pp.1-17 (1993).

43] Seth G.S. and Ghosh, S.K : Ind J. Eng. Sci., V.24, No.7, pp.1183-1193 (1986).

44] Sarkar,D Mukherjee,S:Acta Ciencia Indica.,V.34M,No.2,pp.737-751(2008)

45] Sarojamma,G and Krishna, D.V: Transient hydromagnetic convective flow in a rotating channel with porous boundaries, Acta Mrchanica, V.39, p.277 (1981)

46] Sato,H:J.Phy.Soc.,Japan,V.16,p.1427(1961)

47] Seth,G.S,Ansari,S and Ahmad,N : Acta Ciencia Indica,V.34M, No.4, p.1849 (2008)

48] Sherman,A and Sutton,G.W:Mhd ,Evanston,Illionis,p.173(1961)

48a] Sivaprasad, R, Prasada Rao, D.R.V and Krishna,D.V: Hall effects on unsteady Mhd free and forced convection flow in a porous rotating

49] Singh, K.D and Mathew : An oscillatory free convective MHD flow in a rotating vertical porous channel with heat sources. Ganita, V.60, No.1, pp.91-110 (2009).

50] Sivaiaih, $S$ : Thermo diffusion effects on convective heat and mass transfer through a porous medium in ducts. Ph.D Thesis, SKU, Anantapur, India (2004).

51] Sreevani,M : Mixed convective heat and mass transfer through a porous medium in channels with dissipative effects, Ph.D thesis, S.K.University, Anantapur, India (2002).

52]. Sreerangavani,K,Rajeswara Rao,U and Prasada Rao,D.R.V:Effect of thermo-diffusion on Mhd convection heat and mass transfer flow of chemically reacting fluid through a porous medium in a rotating system.,Presented in $2^{\text {nd }}$ International conference on Applicatrions of Fluid dynamics,July,(2014) 
53] Vijaya Bhaskar Reddy, $\mathrm{P}$ : Mixed convective heat and mass transfer through a porous medium in a horizontal wavy channel with radiation effect. Journal of pure and applied physics, V.22, No.3, (2009).

54] Yamanishi,T:Hall effects on hydromagnetic flow between two parallel plates.,Phy.Soc.,Japan,Osaka,V.5,p.29(1962)

55] Yen, J.I : Effects of wall conductance on Magneto hydrodynamic heat transfer channel. J. Heat transfer. Trans, ASME, V.85C, pp. 371-377 (1973).

56] Zanchini, E : Effect of viscous dissipation on mixed convection in a vertical channel with boundary conditions of the third kind. Int. J. of Heat and Mass Transfer, V.41, pp.3949-3959 (1998).

57]. Sukanya J.S and Leelarathnam A : "combined influence of Hall Currents and Soret effect on convective heat and mass transfer flow past vertical porous stretching plate in rotating fluid and dissipation with constant heat and mass flux and partial slip", IJATED (2018)

58] Jayasudha J.S. and Siva Prasad R : "Soret and Dufour effect on convective heat and mass transfer flow past a vertical porous plate in a rotating fluid with chemical reaction, radiation absorption and dissipation", Ph.D. Thesis, Sri Krishnadevaraya University, Anantapuramu (2017) 